\title{
CONCEPTUAL DESIGN FOR ASSEMBLY IN AEROSPACE INDUSTRY: A METHOD TO ASSESS MANUFACTURING AND ASSEMBLY ASPECTS OF PRODUCT ARCHITECTURES
}

\author{
Bouissiere, Francois (2); Cuiller, Claude (2); Dereux, Pierre-Eric (2); Malchair, Corentin (2); \\ Favi, Claudio (1); Formentini, Giovanni (1) \\ 1: University of Parma; 2: Airbus S.A.S.
}

\begin{abstract}
In recent years, the air transport market has quickly grown, creating new civil aircrafts demand, challenging the actual production rate of aerospace industries. The bottleneck of the current civil aircrafts production rate lies in the capability of the manufacturing and assembly facilities in relation to the aircrafts architecture design.

The aim of this work is to develop a methodology and a related mathematical model that can be used at the conceptual design phase for the assessment of criticalities related to the product assemblability. The methodology allows to recognize modules and/or interfaces which are mostly affecting the assembly time providing a design tool for the comparison and evaluation of product architecture alternatives.

A preliminary application has been done on the nose-fuselage of a civil aircraft for passenger transport. The test case provides interesting outcome in the identification of modules and module interfaces which are strongly affecting the assembly phase and required a re-arrangement (new architecture design) for the process improvement.
\end{abstract}

Keywords: Conceptual design, Product architecture, Design for X (DfX), Aerospace industry, Product manufacturing and assembly

Contact:

Formentini, Giovanni

University of Parma

DIA (Engineering and Architecture Department)

Italy

giovanni.formentini@unipr.it

Cite this article: Bouissiere, F., Cuiller, C., Dereux, P.-E., Malchair, C., Favi, C., Formentini, G. (2019) 'Conceptual

Design for Assembly in Aerospace Industry: A Method to Assess Manufacturing and Assembly Aspects of Product Architectures', in Proceedings of the 22nd International Conference on Engineering Design (ICED19), Delft, The Netherlands, 5-8 August 2019. DOI:10.1017/dsi.2019.303 


\section{INTRODUCTION}

In recent years, the rise of global marketplace has presented an entirely new set of challenges for manufacturing companies. The development of a globally integrated supply chain can lead to extreme advantages to companies which incorporate the proper technology to maximize their return on investment and mitigate their risks. In the case of aerospace industries, focus of the resources is on innovation and business strategy while outsourcing more labour-intensive projects to countries which can produce necessary components at lower costs. In this context, it is required to have an efficient assembly process in order to satisfy the market requests (e.g. time to market, lead time for delivery, etc.). If on one side the request from the market is continuously growing, on another side the manufacturing/assembly lines of civil aircraft shall increase their efficiency in order to deal with these new challenges. A different arrangement of the manufacturing/assembly facilities, together with the adoption of new technologies are hardly achievable due to the extremely high-cost for this complex system. Indeed, it is necessary to work at the (aircraft) product architectural level, trying to improve the design concept to make it more suitable for the assembly phase. Improvements for the assembly phase can be obtained working at the conceptual design level where the degrees of freedom are higher, allowing to find an optimized product architecture towards the assembly process, as seen from the literature analysis on the engineering design process and product development process (Pahl and Beitz, 2007).

Design process is a concrete course of actions for the design of technical systems that derives its knowledge from design science and cognitive psychology, and from practical experiences in different domains (Ulrich and Eppinger, 2011). The design process includes plans of actions that link working steps and design phases according to content and organization. It also includes strategies, rules and principles to achieve general and specific goals as well as methods to solve individual design problems or partial tasks (Howard et al., 2008). The main work on formalization of product development process has been proposed by Pahl and Beitz (2007) in which the product development process has been divided in four (4) phases: (i) Planning and task clarification, (ii) Conceptual design, (iii) Embodiment design, and (iv) Detail design. During the Conceptual design phase, the design process is rapid and interactive and most of the decisions made at this stage will impact the final product cost from a manufacturing and assembly point of view (Zhang at, 2016), thus it is necessary to optimize the product at this phase in order to avoid any excessive increase in the final product cost (Boothroyd et al., 2010). The most widespread tool developed for the formalization of product functions at the conceptual level is the functional analysis of the product (Pahl and Beitz, 2007; Otto and Wood., 1998; Schuh et al., 2014) together with the module heuristics (Stone et al., 2000) to define functional modules. Manufacturing/assembly costs are mostly determined during the design stage, in fact decision made at this stage tends to affect the selection of materials, machine tools and human resources that will be used in the production process (Mikkola, 2007). A variety of methods have been developed to optimize the design process called Design for X (DFX). Among all, Design for Assembly (DFA) methodology provides systematic procedure to maximise use of same components and identify main problematics solution in terms of assembly lead time (Favi et al., 2017). The main drawback of DFA techniques is the applicability: they can be applied only during the Detail design phase, where most of choices have been already made (Lefever and Wood, 1996). Stone et al. (2004) provide a solution by defining a conceptual DFA method to support product architects at the conceptual design phase. The method allows to identify different solutions at conceptual stage and to choose the one that minimizes the assembly/manufacturing cost. Two drawbacks can be identified in this method: (i) the lack of an assembly sequence that minimizes the assembly time (Bedeoui et al., 2018) and (ii) the limited applicability, due to the requirements and needs of different industries (Demoly et al., 2010). An attempt to overcome these restrictions has been made by Favi and Germani (2012). The proposed method allows to identify a proper assembly sequence based on the interface analysis, but it is not applicable to complex product, in which the number of components and interfaces is high, and it does not provide a comparison tool for different product architectures.

The necessity of working at product architectures level is especially important in the aerospace industry in which the development of products requires a very long process compared to the evolution of the market. Indeed, it presents incredible difficulties to adapt products to market request (Polacsek et al., 2017). During years, many attempts have been done to reduce the required process, without any substantial results. In the work of Polacsek et al., (2017) the overall cost of the product is reduced by 
diminishing the lead time, using Resource Constrained Shortest Paths (RCSP) optimization applied to production assembly sequences, correlated / linked to product architecture. Results obtained showed that a co-engineering approach presents many advantages, but it is also time-consuming, and it may require high-skilled operators to run all the necessary analysis. Another attempt has been done by $\mathrm{Li}$ and Lockett, (2017) focusing on the relation between aircraft systems and final assembly stage. Unfortunately, the proposed method lacks design information at the right time for the early design and it faces different challenges such as the complexity of modelling the system behaviour. Thus, there is no generalized method for applying the DFA concept. For complex products, such as aircraft, an enormous number of functions and different constraints (e.g. safety issue, etc.) are present, thus the total degrees of freedom for modules creation are limited and the assembly process becomes extremely important to improve production efficiency.

The aim of this paper is to provide a methodology for the definition of complex product architectures at the conceptual stage, that can be used to assess and compare different architectures from an assemblability point of view. This approach fits with the main issues observed at the manufacturing/assembly department of aircraft industries, addressing all the aspects related to these specific products (e.g. components shape, limited areas to perform assembly operations, complexity of the assembly process, limited accessibility etc.). The methodology is divided in two parts: (i) architecture assembly assessment and, (ii) architecture DFA. This paper is focusing on the first part (i.e. architecture assembly assessment). Within the proposed methodology different domains have been defined (i.e. group of information related to specific aspects) and the collected information are translated into numerical parameters and rules. These parameters are managed through a MultiCriteria-Decision-Making method and a mathematical algorithm to obtain a single assessment score, ranging from zero (0) to one (1), for each module inside the product. The scoring supports architects and designers during product architecture definition, with the focus on the production and assembly. This work can be considered the first attempt of "supporting framework for conceptual design in aerospace" that involves all technical departments related to the product development process (e.g. architecture, design, manufacturing, assembly). The proposed methodology has been developed and tested on a highly complex system that is the nose-fuselage of a civil Airbus aircraft. Interesting outcomes have been retrieved: (i) to assess, in a mathematical way, the assembly performances of a complex system without knowing the assembly lead time (which is a detail design information) and, (ii) to highlight product weaknesses at the architectural level concerning the assembly.

After this introduction, Section 2 presents the proposed approach in detail including the main steps for its development. An Airbus aircraft is analysed as a relevant case study in Section 3. Results are discussed in Section 4 and concluding remarks and future perspectives are highlighted in Section 5.

\section{MATERIALS AND METHODS}

The method is based on a mathematical model developed to consider assembly aspects in the Conceptual design phase. In the Conceptual design phase, it is not possible to have detailed information such as the assembly time for each single assembly task, thus the developed mathematical model should be independent from this information. Indeed, the developed assessment model considers different aspects that affect the assembly time without having directly the assembly time information. These aspects are: (i) parts that need to be installed (i.e. components), (ii) operators performing actions (i.e. assembly process) and, (iii) zones in which operators are working (i.e. ergonomic aspects).

The three aspects can be grouped in three domains:

- Components - It describes modules and/or interfaces that need to be brought and mount inside the product. In order to describe different aspects of this domain, many attributes can be defined.

- Assembly process - It describes the complexity of the assembly operation. Different attributes can be considered to describe this domain, according to the product under analysis.

- Ergonomic aspects - It describes the relation between the area in which operators perform the action and the human factors.

The methodology requires some definitions to be fully understood:

- Module - It represents a group of functions derived by the functional analysis/module heuristics which are translating into an aggregation of physical components/equipment by the means of design options. 
- Equipment - It refers to sub-elements inside the module; a group of equipment can create a module.

- Interface - It describes a functional link between two modules or a module and an aircraft area. It refers to a specific type (derived from Stone):

- Air - Interface between modules regarding air (i.e. air ducts).

- Electrical - Interface between modules regarding electricity (i.e. electrical harnesses).

- Liquid - Interface between modules regarding fluid (i.e. fluid pipes).

- Mechanical - Interface between modules regarding mechanical aspects (i.e. mechanical fasteners, etc.).

- $\quad$ Area - It represents a confined space of the product.

- Domain - It refers to a group of information for specific aspects (e.g. Information regarding ergonomics aspects are grouped into the "Ergonomic domain").

- Attribute - It describes a group of information inside a domain (e.g. the attribute length of harness describes the length of the electrical interface inside the domain "Components").

The proposed methodology consists of seven (7) steps, divided into two main phases: (i) architecture assembly assessment and, (ii) architecture DFA (Figure 1). In the following sub-section only the architecture assembly assessment is analysed and considered. The second phase concerns the way to use results from the assessment phase to drive the definition of new architectures. The second phase will be characterized by the proposed method in two different ways: (i) analyse the reason behind the assessment of "high" (i.e. bad) scores for some interfaces (avoid specific issues related to "high" scores), and (ii) define design rules that can be putted in place to re-build new architectures (avoiding "high" scores).

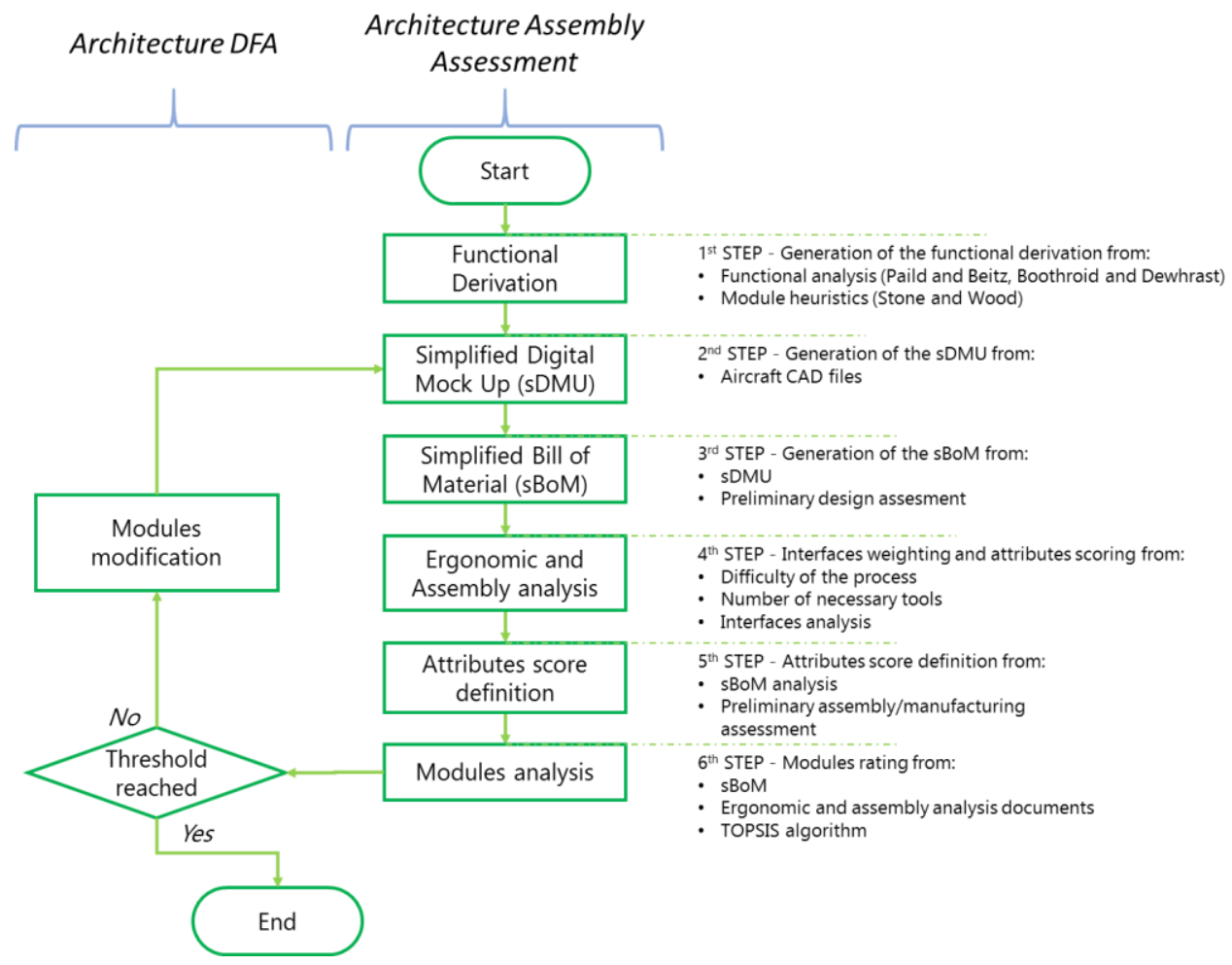

Figure 1. cDFA workflow

\subsection{Functional derivation}

By adopting the functional analysis (Stone et al., 2000), the product functions are obtained. Then, it is necessary to identify basic materials and energy flows (called functional flows) in a graph, using different colours to create a unique correlation between types of functional flows and colour. This will help the user to avoid any possible mistake. The functional flows are obtained by using the classical Pahl et al. (2007) theory. Once the functional derivation has been obtained following the literature (Stone et al., 2000), modules heuristics are applied to derive functional modules. The obtained modules can be: (i) completely new modules, (ii) partially new modules, or (iii) already existing modules (i.e. already implemented inside the product architecture). If modules are new ones, then they are created according to the module heuristics while if they have been already identified the module 
heuristics does not add any value; on the other hand, if some functions partially overlap with the identified not-modifiable modules then it is necessary to adjust the output of the heuristics accordingly to avoid overlapping functions. The final representation obtained is called Functional Representation (Figure 2 - left).

\subsection{Simplified digital mock-up}

The second step consists in the representation of modules in a virtual environment (CAD tool). The Digital Mock-Up (DMU) is a virtual representation of the product that is used mostly during the detailed design phase. Generally, considering the Conceptual design phase, the DMU is too detailed, thus a simplified version is needed, called Simplified Digital Mock-Up (sDMU). The sDMU is a graphical and geometrical representation of a specific architecture related to the system of interest (i.e. aircraft). The sDMU is characterized by simple 3D geometrical items (boxes, cylinder, etc.) describing how functions and flows are connected (Figure 2 - right). The sDMU is the architectural representation of the product under study, enriching the Functional Representation in the physical and geometrical context. Indeed, it presents more detailed information such as the distribution of modules inside the system of interest (module position), the overall module shape and volume (module bounding box), the working area identified for the system of interest and the features of interfaces (e.g. length, shape).
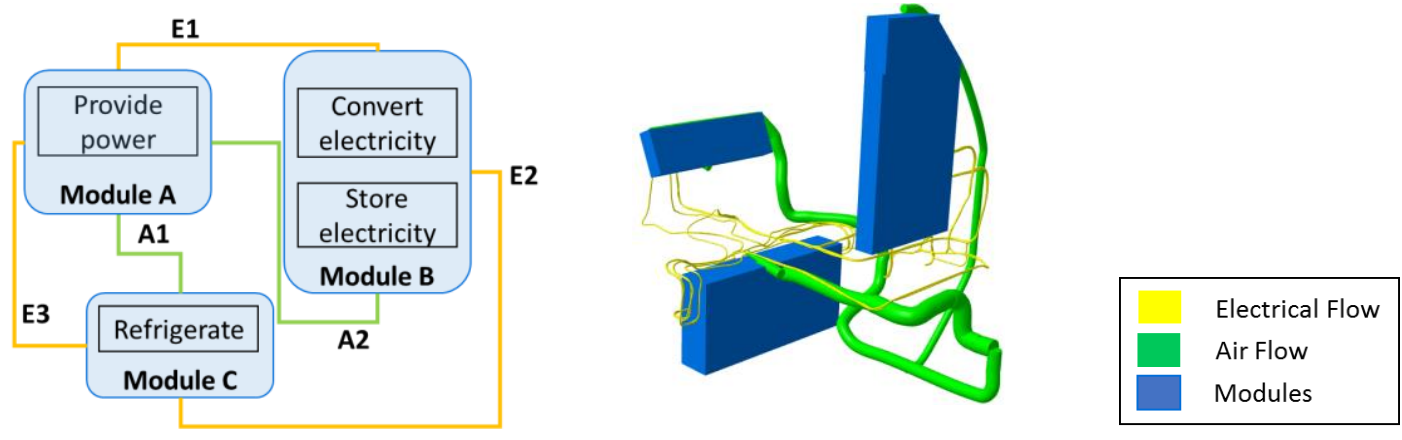

Figure 2. Differences between functional representation (left) and sDMU (right)

The sDMU level of detail and its granularity evolves along the application of the methodology. Indeed, the sDMU is enriched once the method is moving towards a more detail phase of the project, increasing its level of granularity (i.e. adding detail information). The starting version of the sDMU can be obtained by performing a preliminary analysis of the product under study, allowing an estimation of the dimension of modules and/or interfaces to obtain a more realistic result. In this paper, the case study is limited to a sub-set of the nose-fuselage (i.e. only the installation system is analysed such as wires, pipes, shelved, etc.), indeed the remaining part of the aircraft (i.e. nose-fuselage airframe and the rest of the fuselage) is considered "external" to the system of interest. In the $s D M U$ the external constraints derived by the product under study are available such as the main structure of the aircraft i.e. skeleton). If the methodology is used to perform the assessment of an existing product of which a DMU is already available, it is possible to simplify the DMU by neglecting all details and representing modules as boxes with their bounding box and interfaces as cylinder with the real dimension of the connection (Figure 3).
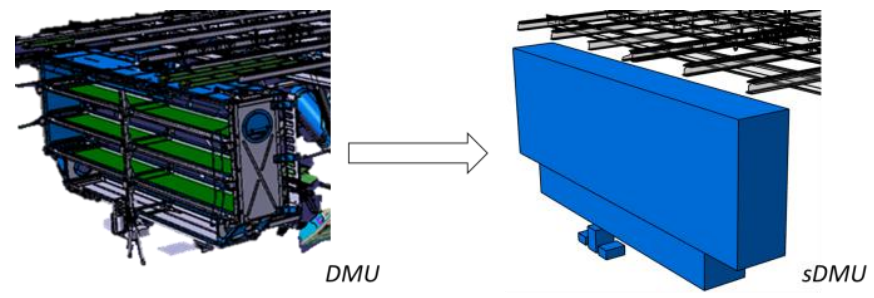

Figure 3. From DMU to $s D M U$

\subsection{Simplified bill of material}

By using the sDMU, a simplified Bill of Material $(s B o M)$ document is created. The $\mathrm{sBoM}$ is a document which translates geometrical and non-geometrical information described by the sDMU into a numerical form used for the mathematical assessment. The $s B o M$ document contains: (i) the information regarding interfaces and how modules are connected and, (ii) the attributes of each interface (e.g. length, etc.) 
which can be defined by the sDMU analysis. The $s B o M$ document groups all the data related to the Components domain. The structure of the $s B o M$ document is presented in Figure 4 - a where Name is a progressive labelling of the interfaces, Subsection is a progressive labelling for a given interface, indicating the working areas crossed by this interface, Type is the interface type (e.g. electric, air), Module IN is the module where the interface starts, and Module OUT is the module where the interface ends. This first part of the sBoM is the same also for the ergonomic analysis and assembly analysis documents.

\subsection{Ergonomic and assembly analysis}

The definition of ergonomic aspects (i.e. ergonomic aspects domain) and product-related assembly aspects (i.e. assembly aspects domain) is described by two documents: (i) the ergonomic analysis document and, (ii) the assembly analysis document. Concerning the ergonomic analysis document, information regarding areas and modules arrangement inside areas is textual. Concerning the assembly analysis document, information regarding the complexity of the assembly operations is textual. The general layout of the ergonomic analysis document and assembly analysis document are presented in Figure $4-\mathrm{b}$ and Figure 4 - c.

First it is necessary to consider the interfaces of interest then the attributes that describe each interface. The interfaces have to be the same for each document while the number of attributes can vary according to the interface and the document, meaning the number of columns for each interface, in each document, can vary.

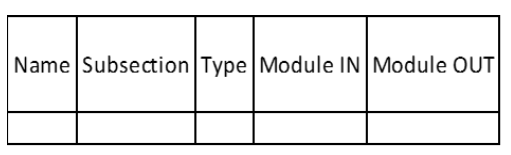

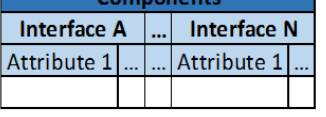

(a)

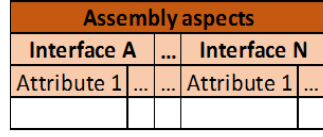

(b)

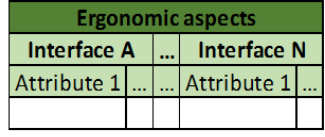

(c)

Figure 4. sBoM (a), assembly aspect Document (b), ergonomic aspect Document (c)

\subsection{Attributes score definition}

Since the data (both numerical and textual) reported in the three documents are heterogeneous, they require to be normalized for the assessment and comparison. A possible method to normalize the data is to create a ranking scale (e.g. from one (1) to five (5)). Scoring matrices need to be defined to switch from numerical values and textual information to normalized scores. It is worth noting that this step is an important adding value of the methodology due to the following reasons: (i) it allows to map the industrial know-how, collecting information from manufacturing and assembly department, onto the assessment of critical modules and interfaces, (ii) it defines design guidelines oriented to product manufacturing and assembly. Indeed, the collected knowledge can be reinjected into the second phase of the proposed design methodology (architecture DFA) for the definition of new optimized product architectures. Different approaches can be used to define ranges for the scoring matrices, according to the document analysed:

- $\quad \mathrm{sBoM}$ - Since it is composed only of numerical values, it is possible to perform a statistical analysis to obtain the scoring matrix (Figure 5 - a).

- $\quad$ ergonomic analysis document and assembly analysis document - These two documents present only textual information assessed and defined with the help of the manufacturing department (Figure 5 - b).

\begin{tabular}{|r|l|}
\hline Score & Attribute A - Range \\
\hline 1 & Below value $A$ \\
\hline 2 & From value $A$ to value $B$ \\
\hline 3 & From value $C$ to value $D$ \\
\hline 4 & From value $E$ to value $F$ \\
\hline 5 & Over value $F$ \\
\hline
\end{tabular}

(a)

\begin{tabular}{|r|l|}
\hline Score & Attribute B - Range \\
\hline 1 & Area A \\
\hline 2 & Area B \\
\hline 3 & Area C \\
\hline 4 & Area D \\
\hline 5 & Area E \\
\hline
\end{tabular}

(b)

Figure 5. General scoring matrix for numerical information (a) and string information (b) 


\subsection{Modules analysis}

Module analysis is the last step of the methodology and allows to obtain the final "assembly score" for each module that characterizes the product architecture. This step is composed of five (5) phases:

1. The normalization of the values inside $s B o M$ document, ergonomic analysis document and assembly analysis document (by using scoring matrices defined in the previous step).

2. The collection of the normalized values inside the ergonomic analysis document by the means of the Root Mean Square (RMS) operator, obtaining a single score for the ergonomic domain. The choice of the RMS operator is justified by the differences in the attribute's values for the "ergonomic" domain. Since errors are squared before been averaged, the RMS gives a relatively high weight when values are very different from each other. Therefore, the RMS is suitable when large errors are particularly undesirable and there is a random distribution of data.

3. The collection of the normalized values inside the assembly analysis document using the Mean operator (MEAN), obtaining a single score for the assembly domain. The choice of the MEAN operator is justified by the differences in the attribute's values for the "assembly" domain. In fact, they are different but with a close meaning, indeed their values do not change randomly.

4. The merging of the normalized values inside the $\mathrm{sBoM}$ with the single score obtained for ergonomic analysis document and assembly analysis document, creating a new document called Extended simplified Bill of Material (EsBoM)

5. The application of the TOPSIS method: (i) to switch from interfaces information (i.e. EsBoM) to modules information and (ii) to obtain a single score for each module ranging from zero (0) to (1), the higher the score, the more critical the module is in terms of assemblability.

To perform the 5th phase, among all the Multi-Attribute-Decision-Making (MADM), the TOPSIS has been chosen for its easiness of implementation and the possibility to deal with more parameters without need of modifications (Valasquez and Hester, 2013). The workflow of the module analysis phase is shown in Figure 6.

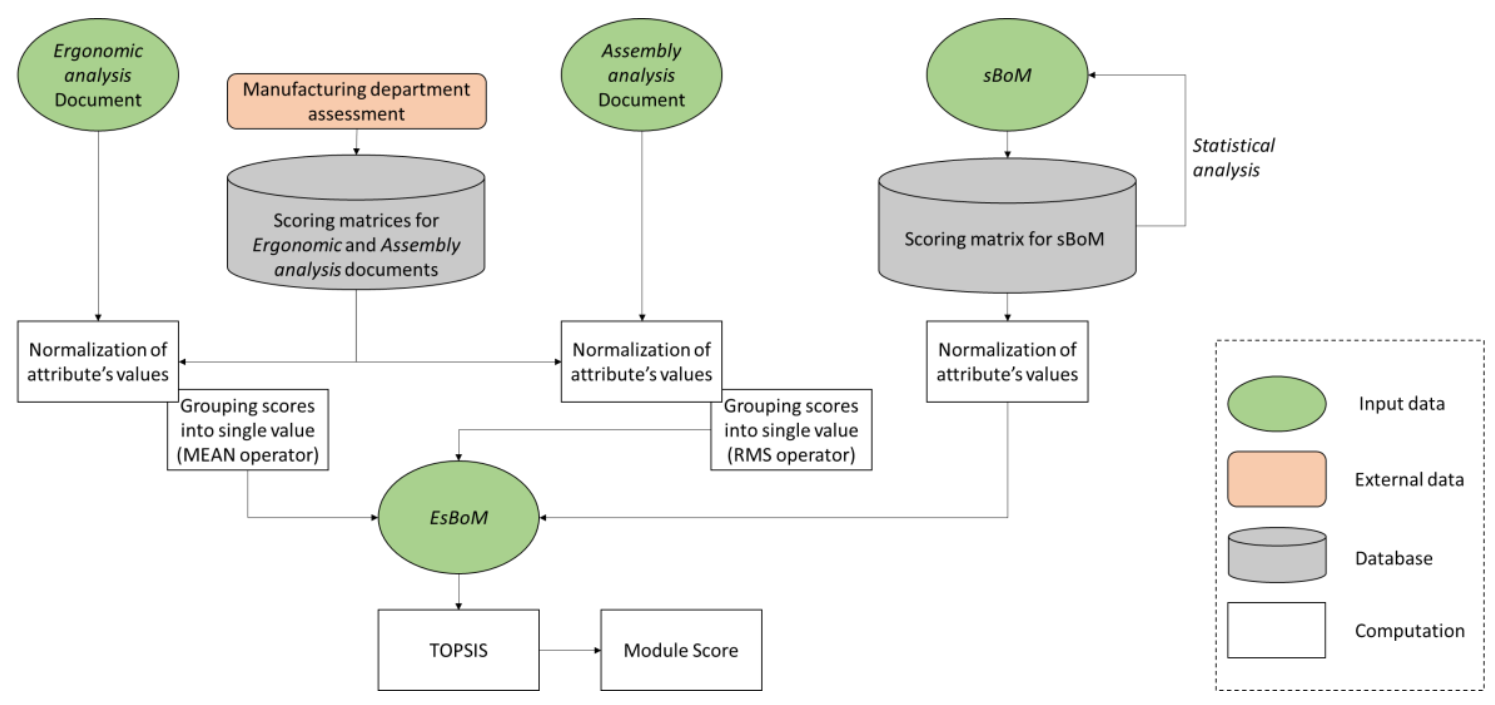

Figure 6 - Module analysis workflow

\section{CASE STUdY}

The developed methodology has been applied on a simplified version of the nose-fuselage of an Airbus aircraft. The nose-fuselage is a highly complex system of the overall aircraft, including several modules and equipment assembled in a very constrained environment. These aspects lead the case study to be an adding value for the proposed method which includes the mapping of industrial know-how starting from the most complex aircraft system and the assessment of critical modules and interfaces. In particular, the present case study aims to demonstrate how to collect manufacturing/assembly knowledge on an existing product for the subsequent exploitation of results that will be used to drive the system design evolution toward optimized architecture in terms of assemblability and manufacturability, and, ultimately, to design a completely new aircraft. The methodology has been applied in a simplified system which considers only "electrical" and "air" interfaces. This is a simplification related to the fact that the mentioned interfaces are the most impacting from the assemblability point of view on the currently 
aircraft in production. The methodology has been applied following the steps reported in the methodology section.

Step 1 - Functional derivation: functional representation of the nose-fuselage has been performed and modules have been identify using module heuristics.

Step 2 - simplified Digital Mock Up: the sDMU has been obtained by simplifying an already existing DMU. Modules have been modelled considering: (i) the physical position (x, y, z) inside the nosefuselage (module position), (ii) the module shape and volume (module bounding box), (iii) the manufacturing/assembly working area. Interfaces have been modelled accordingly with the functional representation but providing also: (i) interface length, (ii) interface shape, and (iii) interface position $(\mathrm{x}, \mathrm{y}, \mathrm{z})$ of the connection with the module.

Step 3 - simplified Bill of Material: the attributes considered for the electrical interface in the sBoM (Figure 7) are: (i) length of harness, and (ii) number of connections; while for the air interface: (i) length of duct, and (ii) bends.

\begin{tabular}{|c|c|c|c|c|c|c|c|c|}
\hline \multirow[b]{3}{*}{ Name } & \multirow[b]{3}{*}{ Subsection } & \multirow[b]{3}{*}{ Type } & \multirow[b]{3}{*}{ Module IN } & \multirow[b]{3}{*}{ Module OUT } & \multicolumn{4}{|c|}{ Components } \\
\hline & & & & & \multicolumn{2}{|l|}{ Air } & \multicolumn{2}{|c|}{ Electrical } \\
\hline & & & & & Length of ducts & Bends & Length of harness & Numb. of connections \\
\hline A5 & 5.1 & Air & Module C & Module F & 2,1 & 3 & & \\
\hline & 5.2 & & & & 2 & 3 & & \\
\hline A6 & 6.1 & Air & Module D & Module A & 1,1 & 4 & & \\
\hline & 6.2 & & & & 2 & 5 & & \\
\hline E1 & 1.1 & Electrical & IModule D & Module B & & & 28,2 & 35 \\
\hline & 1.2 & & & & & & 0,5 & 更 \\
\hline
\end{tabular}

Figure 7. Extract of the $s B o M$

Step 4 - Ergonomic and Assembly analysis: for the ergonomic domain the following attributes have been identified: (i) working area which describes the area in which the operator needs to perform the assembly operation, (ii) zone which describes the working area in relation to ergonomic criteria, (iii) access which describes the available access to bring components inside the aircraft, and, (iv) working space size that describes the available volume of the area in which operator performs the assembly action. Regarding the assembly domain, two (2) attributes have been considered: (i) variety of tools that takes in consideration the number of tools necessary to perform the assembly action and, (ii) process which considers the complexity of the assembly process (i.e. need of specialized operators, etc.).

Step 5 - Attributes score definition: the chosen ranking scale for scoring matrices is from one (1) to five (5). The scoring matrix for the sBoM has been obtained through statistical analysis of the values inside the document, while the scoring matrices for ergonomic and assembly domains have been obtained though survey and meeting with the manufacturing department. It is worth noting that the scoring matrices retrieved by the involvement of manufacturing and assembly department created the internal know-how elicitation for the development of the proposed method in one of the most critical assembly. Step 6 - Modules analysis: after the data collection, the CDfA analysis has been performed. Results of this step are presented in Figure 8. The Overall Score refers to the overall value of the interface under study, obtained by means of mathematical operators, before the TOPSIS method is applied. The column TOPSIS Algorithm presents the computational steps typical of the TOPSIS method; values inside the TOPSIS Algorithm column have been hidden for the sake of brevity. Finally, the column SCORE presents the score of each module, ranging from zero (0.0) to one (1.0). The association between score and modules is highlighted using a grey background (e.g. the score 0.42 refers to the Module A, 0.19 to the Module B, etc.). Output shows that Module L, with a score of 0.72, is the most critical from an assembly point of view, in fact it has the highest score among all. The second critical module is the Module E with a score of 0.68 , in which the interface electrical impacts more (3.11) than the air interface (2.81). Following steps of the methodology would target the 2 modules for re-design.

\section{RESULTS EVALUATION}

The obtained results give a clear understanding of the overall situation at the architectural level regarding the expected assembly duration of each module. The obtained results evaluate the complexity of interfaces which are linking different modules. Indeed, the developed numerical model weights more modules that present higher complexity from the interface point of view. For instance, the module $\mathrm{L}$ is the most critical modules because it has a high number of interfaces (both "electrical" and "air"). The 
"electrical" interface presents a score of 3.34, while the "air" interface presents a score of 2.81. The lowest score (0.19) is obtained for the Module A, since it has the lower number of "electrical" and "air" interfaces with respect to all the other modules. In addition, the interfaces connected to the Module A show small lengths in working environments considered less critical from the manufacturing/assembly department. Between module A and module L fall all other modules which have different levels of complexity related to number of interfaces, the features of the interfaces and the associated ergonomic factors addressed considering where the interface is passing through (working environment). The methodology does not assess the complexity of the module itself, in fact the module has (i) an "intrinsic" complexity related to the module features (e.g. shape, dimensions, etc.), and (ii) "external" complexity related to the area (working environment) where the module is installed. This is a limitation of the proposed methodology in its current stage and will be analysed in the future developments.

\begin{tabular}{|l|l|r|c|c|}
\hline Interface & Module & Overall Score & TOPSIS Algorithm & SCORE \\
\hline A & Module A & 2,61 & $\ldots$ & \\
\hline E & Module A & 2,63 & $\ldots$ & 0,42 \\
\hline A & Module B & 1,95 & $\ldots$ & \\
\hline E & Module B & 2,81 & $\ldots$ & 0,19 \\
\hline A & Module C & 2,39 & $\ldots$ & \\
\hline E & Module C & 2,66 & $\ldots$ & 0,30 \\
\hline A & Module D & 2,56 & $\ldots$ & \\
\hline E & Module D & 2,60 & $\ldots$ & 0,38 \\
\hline A & Module E & 2,84 & $\ldots$ & \\
\hline E & Module E & 3,11 & $\ldots$ & 0,68 \\
\hline A & Module F & 2,84 & $\ldots$ & \\
\hline E & Module F & 2,69 & $\ldots$ & 0,48 \\
\hline
\end{tabular}

\begin{tabular}{|l|l|r|c|c|}
\hline Interface & Module & Overall Score & TOPSIS Algorithm & SCORE \\
\hline $\mathrm{A}$ & Module G & 2,77 & $\ldots$ & \\
\hline $\mathrm{E}$ & Module G & 2,81 & $\ldots$ & 0,55 \\
\hline $\mathrm{A}$ & Module H & 2,84 & $\ldots$ & \\
\hline $\mathrm{E}$ & Module H & 2,84 & $\ldots$ & 0,60 \\
\hline $\mathrm{A}$ & Module I & 2,99 & $\ldots$ & \\
\hline $\mathrm{E}$ & Module I & 2,63 & $\ldots$ & 0,58 \\
\hline $\mathrm{A}$ & Module L & 2,81 & $\ldots$ & \\
\hline $\mathrm{E}$ & Module L & 3,34 & $\ldots$ & 0,72 \\
\hline $\mathrm{A}$ & Module M & 2,81 & $\ldots$ & \\
\hline $\mathrm{E}$ & Module M & 2,72 & $\ldots$ & 0,53 \\
\hline $\mathrm{A}$ & Module N & 3,29 & $\ldots$ & \\
\hline $\mathrm{E}$ & Module N & 2,44 & $\ldots$ & 0,60 \\
\hline $\mathrm{A}$ & Module T & 2,93 & $\ldots$ & \\
\hline $\mathrm{E}$ & Module T & 2,71 & $\ldots$ & 0,59 \\
\hline
\end{tabular}

Figure 8. Results of TOPSIS

It is interesting to notice that results have been obtained by considering components, assembly and ergonomic domains. Indeed, the obtained results can be considered significant because take into consideration all aspects involved in the assembly process. Results show many possibilities to architects to improve assemblability (lower the score) such as changing components shape, components connections, operator position or simplifying the process complexity to achieve a lower score.

Two main drawbacks can be identified: (i) the lack of proper guidelines to how perform modification of the architecture and, (ii) the difficulty to compare different results, in fact, the TOPSIS method generates only relative scores, thus, scores can be only lowered with respect to other modules. Moreover, the method has been tested only on one simplified version of a product, thus results may not be robust enough. On the other hand, the chosen case of study is highly complex, giving confidence in its ability to address less complex product.

\section{CONCLUSION}

The provided methodology is proposing a structured way to consider assembly aspects at the Conceptual design phase of a complex product. The outcome of the method provides a useful picture of the overall assembly situation for each module inside the product. Moreover, the method is scalable and can be applied to different complex products (e.g. aircraft, vessels, etc.) by selecting proper interfaces, attributes and tuning the scoring matrices. The main advantages of the developed method are: (i) to provide a single score for each module inside the product, (ii) to help designer to spot weaknesses at architectural level for the assembly procedure and, (iii) to take into consideration at the beginning of the product development process the assembly aspects, considered, in general, during the final stage of the product development process. The following drawbacks of the methodology can be identified by the direct implementation in a real case study: (i) the lack of proper guidelines for modifying the product architecture to improve the product assembly, (ii) the need of large amount of data to fill the documents and, (iii) the difficulty to compare different architecture results (the TOPSIS method generates only relative scores, thus, scores can be only lowered with respect to other modules). The methodology has been tested on a complex system, which provides an interesting test case for the methodology validation. In addition, the present case study provides a good "scalability" of the methodology to less complex product. Finally, the proposed methodology represents one of the first attempt to deliver an assessment 
tool of the assembly time for product architectures, thus it should be considered a starting point toward a new group of methods. Several improvements can be considered. First, clear guidelines to how modify modules should be added, then a method for comparing different product architectures must be investigated. Regarding the last point, a possible solution to compare different product architecture is to consider the Overall Score $(O S)$ instead of the SCORE to compare products architectures. In particular, it is possible to perform the following computation:

$$
\frac{O S(n+1)}{O S(n)} \%=C V
$$

Where $O S(n)$ is the Overall Score of each interface for the product architecture under analysis at iteration $\mathrm{n}$ of the methodology, OS $(n+1)$ is the Overall Score of each interface obtained with a new run of the methodology $(\mathrm{n}+1)$ on a modified product architecture, and $C V$ is the result (i.e. Comparison Value). For CV values smaller than $100 \%$ an improvement on the assembly time has been obtained for the new product architecture, for values greater than $100 \%$ the opposite holds. For CP values equal to $100 \%$, no changes have occurred.

\section{REFERENCES}

Bedeoui, A. et al. (2018), "Assembly plans generation of complex machines based on the stability concept", Procedia CIRP, Vol. 70, pp. 66-71. http://dx.doi.org/10.1016/j.procir.2018.03.030.

Boothroyd, G. (1994), "Product design for manufacture and assembly", Computer-Aided Design, Vol. 26 No. 7 , pp. 505-520. http://dx.doi.org/10.1016/0010-4485(94)90082-5.

Demoly, F., et al. (2011), "An assembly oriented design framework for product structure engineering and assembly sequence planning", Robotics and Computer-Integrated Manufacturing, Vol. 27 No. 1, pp. 33-46. http://dx.doi.org/10.1016/j.rcim.2010.05.010.

Favi, C. and Germani, M. (2012), "A method to optimize assemblability of industrial product in early design phase: from product architecture to assembly sequence", International Journal on Interactive Design and Manufacturing (IJIDeM), Vol. 6 No. 3, pp. 155-169. http://dx.doi.org/10.1007/s12008-012-0147-y.

Favi, C., Germani, M. and Mandolini, M. (2017), "Development of complex products and production strategies using a multi-objective conceptual design approach", The International Journal of Advanced Manufacturing Technology, Vol. 95 No. 1-4, pp. 1281-1291. http://dx.doi.org/10.1007/s00170-017-1321-y.

Howard, T.J., Culley, S.J. and Dekoninck, E. (2008), "Describing the creative design process by the integration of engineering design and cognitive psychology literature", Design Studies, Vol. 29 No. 2, pp. 160-180. http://dx.doi.org/10.1016/j.destud.2008.01.001.

Lefever, D.D. and Wood, K.L. (1996), "Design for assembly techniques in reverse engineering and redesign", In: the Proceedings of ASME Design Theory and Methodology Conference.

$\mathrm{Li}, \mathrm{T}$. and Lockett, H. (2017), "An investigation into the interrelationship between aircraft systems and final assembly process design", Procedia CIRP, Vol. 60, pp. 62-67. http://dx.doi.org/10.1016/j.procir.2017.01.056.

Mikkola, J.H. (2007), "Management of product architecture modularity for mass customization: modeling and theoretical considerations", IEEE Transactions on Engineering Management, Vol. 54 No. 1, pp. 57-69. http://dx.doi.org/10.1109/tem.2006.889067.

Otto, K.N. and Wood, K.L. (1998), "Product evolution: a reverse engineering and redesign methodology", Research in Engineering Design, Vol. 10 No. 4, pp. 226-243. http://dx.doi.org/10.1007/s001639870003.

Pahl, G., Beitz, W., Feldhusen, J. and Grote, K.-H. (2007), Engineering Design, Wallace K., London, http://dx.doi.org/10.1007/978-1-4471-3581-4.

Polacsek, T. et al. (2017), "Towards thinking manufacturing and design together: An aeronautical case study", Lecture Notes in Computer Science, pp. 340-353. http://dx.doi.org/10.1007/978-3-319-69904-2_27.

Schuh, G., Rudolf, S. and Vogels, T. (2014), "Development of modular product architectures", Procedia CIRP, Vol. 20, pp. 120-125. http://dx.doi.org/10.1016/j.procir.2014.05.042.

Stone, R.B., McAdams, D.A. and Kayyalethekkel, V.J. (2004), "A product architecture-based conceptual DFA technique”, Design Studies, Vol. 25 No. 3, pp. 301-325. http://dx.doi.org/10.1016/j.destud.2003.09.001.

Stone, R.B., Wood, K.L. and Crawford, R.H. (2000), "A heuristic method for identifying modules for product architectures", Design Studies, Vol. 21 No. 1, pp. 5-31. http://dx.doi.org/10.1016/s0142-694x(99)00003-4.

Ulrich, K. and Eppinger, S.A. (2011), Product Design and Development, McGraw-Hill Education, New York.

Velasquez, M. and Hester, P. (2013), "An analysis of multi-criteria decision making methods", International Journal of Operations Research, Vol. 10, pp. 56-66.

Zhang, H. at al. (2016), "A new conceptual design method to support rapid and effective mapping from product design specification to concept design", International Journal of Advanced Manufacturing Technology, Vol. 87 No. 5-8, pp. 2375-2389. http://dx.doi.org/10.1007/s00170-016-8576-6. 